\title{
Oscillatory Convection in Rotating Spherical Shells: Low Prandtl Number and Non-Slip Boundary Conditions*
}

\author{
F. Garcia ${ }^{\dagger}$, J. Sánchez ${ }^{\dagger}$, E. Dormy $y^{\ddagger}$, and M. Net ${ }^{\dagger}$
}

\begin{abstract}
A five-degree model, which reproduces faithfully the sequence of bifurcations and the type of solutions found through numerical simulations of the three-dimensional Boussinesq thermal convection equations in rotating spherical shells with fixed azimuthal symmetry, is derived. A low Prandtl number fluid of $\sigma=0.1$ subject to radial gravity, filling a shell of radius ratio $\eta=0.35$, differentially heated, and with non-slip boundary conditions, is considered. Periodic, quasi-periodic, and temporal chaotic flows are obtained for a moderately small Ekman number, $E=10^{-4}$, and at supercritical Rayleigh numbers of order $R a \sim O\left(2 R a_{c}\right)$. The solutions are classified by means of frequency analysis and Poincaré sections. Resonant phase locking on the quasi-periodic branches, as well as a sequence of period doubling bifurcations, are also detected.
\end{abstract}

Key words. thermal convection, rotating flows, spherical geometry, modulated wave solutions, period doubling, low-dimensional model

AMS subject classifications. 35Q86, 37G35, 37N10, 76E06, 76U05

DOI. $10.1137 / 15 \mathrm{M} 100729 \mathrm{X}$

1. Introduction. The study of the thermal convection in rotating spherical geometries is fundamental to explaining many geophysical and astrophysical phenomena, such as the generation of the magnetic fields, or the differential rotation observed in the atmosphere of the major planets. There is a multitude of papers devoted to studying the role of thermal convection in the dynamics of the celestial bodies. Good reviews can be found in the literature; see, for instance, [21] and [29] or the introductions of [6] and [19]. The difficulties related to the experimental studies in these fields enhance the importance of three-dimensional numerical simulations. For this reason the development and improvement of the numerical techniques is basic for this research.

Nowadays the onset of convection in spherical shells is well described and understood mainly with the help of numerical simulations $[9,1,28,17]$ and asymptotic theories $[33,5,37$, 9, 39, 41, 4]. Convection usually arises as rotating waves (thermal Rossby waves) traveling

\footnotetext{
${ }^{*}$ Received by the editors February 6, 2015; accepted for publication (in revised form) by D. Barkley July 29, 2015; published electronically October 20, 2015.

http://www.siam.org/journals/siads/14-4/M100729.html

${ }^{\dagger}$ Dept. de Física Aplicada, Universitat Politècnica de Catalunya, Campus Nord, Mòdul B4, Jordi Girona Salgado 1-3, 08034 Barcelona, Spain (fernando.garcia-gonzalez@upc.edu, Juan.J.Sanchez@upc.edu, marta.net@upc.edu). This research was supported by MEC-DGICT/FEDER project FIS2013-40674-P and AGAUR-GENCAT project 2014SGR-1145. The work of the first author was also supported by the Fondation Sciences Mathématiques de Paris (FSMP) and by a public grant overseen by the French National Research Agency (ANR) as part of the Investissements d'Avenir program (reference: ANR-10-LABX-0098). This work was granted access to the HPC resources of MesoPSL financed by the Region Ile de France and the project Equip@Meso (reference ANR-10-EQPX-29-01) of the programme Investissements d'Avenir supervised by the Agence Nationale pour la Recherche.

${ }^{\ddagger}$ MAG, LRA, Département de Physique, Ecole Normale Supérieure, 24 rue Lhomond, 75005 Paris, France (dormy@phys.ens.fr).
} 
in the azimuthal direction, which break the invariance of the basic conduction state. The difficulty of solving satisfactorily the full three-dimensional problem has led to alternative ways of study. One is to use two-dimensional models (quasi-geostrophic) [3, 27, 32, 19] by applying a $z$-averaging technique; another way is to calculate three-dimensional solutions of symmetry and/or fixed parity to achieve parameter values as high as possible. However, quasi-geostrophic models cannot be used for rotating spherical convection with very small Prandtl numbers. New linear and weakly nonlinear quasi-geostrophic approximations were developed in [25]. They are valid in spherical geometry and fulfill the mass conservation, since they allow one to determine the radial extension of the convective motions depending on the Prandtl number, and the horizontal components of the velocity field are not simply defined by a stream function.

A common practice in problems described by PDEs is to obtain reduced theoretical models from the information supplied by the numerical simulations, to explore the problem in greater depth. For instance, in the context of magnetohydrodynamics, an amplitude equation was derived in $[31,30]$ by expanding the magnetic field in terms of two axisymmetric stationary modes, providing a simple explanation of the reversals of Earth's magnetic field as well as of the dynamical regimes of the magnetic field generated by the dynamo effect in the von Kármán sodium experiment. The book by Chossat and Iooss [7], dedicated to the CouetteTaylor problem, contains much information on the application of center manifold reduction and bifurcation theory in the presence of symmetries to obtain amplitude equations in several situations, multicritical points, imperfections, amplitude modulations, etc. Examples in thermal convection can be found in [24,35], among many others. In the first paper, a center manifold reduction and normal form theory were used to deduce a low-dimensional system of ODEs, reproducing the full local behavior of the PDEs around codimension-two double-Hopf points, where the transition between axisymmetric steady solutions and nonaxisymmetric rotating waves occurred. In [35] the amplitude equations and coefficients describing the dynamics close to a triple- $(+1)$ bifurcation of a numerically computed $\mathbb{D}_{4}$-symmetric periodic orbit were obtained from the symmetries of the eigenfunctions for a thermal convection problem with $\mathbb{O}(2)$ symmetry.

Most of the full three-dimensional studies of the first stages of convection in spherical geometry are based on direct numerical simulations, mainly with stress-free boundary conditions (see $[42,2,38,8,36,11]$, among many others), some of them to avoid solving the thin Ekman boundary layers that appear when the non-slip condition is imposed. In this case the numerical simulations require higher spatial resolutions and thus are computationally most demanding. From a physical point of view, stress-free boundary conditions are appropriate for modeling planet atmospheres, while non-slip boundaries are more adequate for the study of the dynamics of the Earth's fluid core and for comparison with laboratory experiments. Moreover, rigid boundaries slow down the fluid inhibiting the generation of mean zonal flows and enhance the growth of angular momentum and vorticity by means of the walls. The above-mentioned references focused on the study of the physical properties of each type of oscillatory solution found at different regions of the parameter space. For instance, in [42] it was found that for $\sigma=\infty$ the instability of the traveling waves gives rise to amplitude or shape modulations. Several types of oscillations with different azimuthal wave numbers were found at low $\sigma$ in [2]. In both cases the authors found a strong increase of the heat transfer

Copyright (c) by SIAM. Unauthorized reproduction of this article is prohibited. 
produced by the onset of quasi-periodic flows. A more detailed study of the finite-amplitude physical properties, including helicity or differential rotation, and of the role of the equatorial symmetry or the onset of polar convection is performed in [38] for several Prandtl numbers. With $\sigma=1$ and for weak supercritical conditions the flow is strongly oscillatory, displaying an intermittent pattern [8]. The sequence of transitions observed in the full three-dimensional simulations of [36] was reproduced in [26] by using the quasi-geostrophic approximation. The authors found that, as the Ekman number is decreased, the transition to oscillatory convection occurs for marginally supercritical Rayleigh numbers.

Recently, several authors have tried to understand the transitions among flows by applying bifurcation theory, commonly employed in ODEs. For instance, the multiplicity of stable patterns of rotating waves, their transitions to modulated waves, and their classification according to their spatio-temporal symmetry have been studied in [11] at moderate $E$ by means of direct numerical simulations. The bifurcation diagrams and the stability of the traveling waves in the slowly rotating regime, for $\sigma=1$, were studied in [22] with non-slip boundary conditions by means of Newton's method. At lower $E$ and $\sigma$, which require higher spatial resolutions, Newton-Krylov continuation techniques and Arnoldi methods were applied successfully in [34] to explain the coexistence of stable traveling waves due to the presence of a double-Hopf bifurcation, and to understand the existence of amplitude- and shape-modulated waves from the symmetry breaking of the eigenfunctions at the secondary bifurcations.

The results presented here shed light on the type of bifurcations existing between the oscillatory flows described in the preceding numerical studies for non-slip boundary conditions and Prandtl number $\sigma=0.1$. The system is integrated by fixing the azimuthal wave number $m$, and, to have accurate solutions, semi-implicit backward differentiation-extrapolation formulas (IMEX-BDF) implemented with variable step size and variable order are used. In this way it has been possible to obtain unstable solutions by just time integration. The study of branches of unstable traveling and modulated waves with fixed azimuthal wave number is important because they drive the dynamics of the full three-dimensional model. For instance, the intermittent solutions shown in [34] can be understood in terms of a heteroclinic chain which connects the two unstable solutions with different azimuthal symmetry $(m=4,5)$ studied here. In addition, the bifurcation diagrams presented here serve to explain the jumps between branches and the appearance of more complex attractors, obtained without symmetry constraints, such as three-frequency quasi-periodic flows found in [13] when the Rayleigh number is increased from that of the onset of convection.

Following the above-mentioned line of investigation, and aside from the use of non-slip boundary conditions and low $\sigma$ and $E$ values, the main goal of this study is to try to understand the oscillatory bifurcations of a large-scale system in the context of dynamical systems theory, building empirically a simple system of three ODEs (two of them complex and one real) that is able to capture the transitions between the periodic orbits and attracting two-tori together with their posterior period doublings that lead to chaotic solutions.

The paper is organized as follows. In section 2 we introduce the formulation of the problem, and the numerical method used to obtain the solutions. In section 3 the bifurcation diagrams as a function of $R a$, the frequency spectrum, and Poincaré sections of the convective flows are analyzed. The patterns of the oscillatory type of convection are shown in section 4 . The derivation of a reduced model and the comparison of its solutions with those of the three-

Copyright (c) by SIAM. Unauthorized reproduction of this article is prohibited. 
dimensional equations are performed in section 5. Finally, in section 6 the paper ends with a brief summary of the results obtained.

2. Mathematical model and numerical integration. We consider the thermal convection of a fluid filling the gap between two concentric spheres differentially heated, rotating about an axis of symmetry with constant angular velocity $\boldsymbol{\Omega}=\Omega \mathbf{k}$, and subject to radial gravity $\mathbf{g}=-\gamma \mathbf{r}$, where $\gamma$ is constant and $\mathbf{r}$ is the position vector. The mass, momentum, and energy equations are written in the rotating frame of reference. The units are $d=r_{o}-r_{i}$ for the distance, $\nu^{2} / \gamma \alpha d^{4}$ for the temperature, and $d^{2} / \nu$ for the time. In the previous definitions $r_{i}$ and $r_{o}$ are the inner and outer radii, respectively, $\nu$ is the kinematic viscosity, and $\alpha$ is the thermal expansion coefficient.

We use the Boussinesq approximation, and the solenoidal velocity field is expressed in terms of toroidal, $\Psi$, and poloidal, $\Phi$, potentials:

$$
\mathbf{v}=\boldsymbol{\nabla} \times(\Psi \mathbf{r})+\nabla \times \nabla \times(\Phi \mathbf{r}) .
$$

Consequently, the equations for both potentials, and the temperature perturbation, $\Theta=$ $T-T_{c}$, from the conduction state $\mathbf{v}=\mathbf{0}, T=T_{c}(r)$, with $r=|\mathbf{r}|$, are

$$
\begin{aligned}
{\left[\left(\partial_{t}-\nabla^{2}\right) L_{2}-2 E^{-1} \partial_{\varphi}\right] \Psi } & =-2 E^{-1} \mathcal{Q} \Phi-\mathbf{r} \cdot \boldsymbol{\nabla} \times(\boldsymbol{\omega} \times \mathbf{v}), \\
{\left[\left(\partial_{t}-\nabla^{2}\right) L_{2}-2 E^{-1} \partial_{\varphi}\right] \nabla^{2} \Phi+L_{2} \Theta } & =2 E^{-1} \mathcal{Q} \Psi+\mathbf{r} \cdot \nabla \times \nabla \times(\boldsymbol{\omega} \times \mathbf{v}), \\
\left(\sigma \partial_{t}-\nabla^{2}\right) \Theta-\operatorname{Ra} \eta(1-\eta)^{-2} r^{-3} L_{2} \Phi & =-\sigma(\mathbf{v} \cdot \boldsymbol{\nabla}) \Theta
\end{aligned}
$$

where $\boldsymbol{\omega}=\boldsymbol{\nabla} \times \mathbf{v}$ is the vorticity.

The parameters of the problem are the Rayleigh number $R a$, the Prandtl number $\sigma$, the Ekman number $E$, and the radius ratio $\eta$. They are defined by

$$
R a=\frac{\gamma \alpha \Delta T d^{4}}{\kappa \nu}, \quad E=\frac{\nu}{\Omega d^{2}}, \quad \sigma=\frac{\nu}{\kappa}, \quad \eta=\frac{r_{i}}{r_{o}},
$$

where $\kappa$ is the thermal diffusivity and $\Delta T>0$ is the difference in temperature between the inner and outer boundaries.

The operators $L_{2}$ and $\mathcal{Q}$ are defined by $L_{2} \equiv-r^{2} \nabla^{2}+\partial_{r}\left(r^{2} \partial_{r}\right), \mathcal{Q} \equiv r \cos \theta \nabla^{2}-\left(L_{2}+\right.$ $\left.r \partial_{r}\right)\left(\cos \theta \partial_{r}-r^{-1} \sin \theta \partial_{\theta}\right),(r, \theta, \varphi)$ being the spherical coordinates, with $\theta$ measuring the colatitude and $\varphi$ the longitude. When non-slip perfect thermally conducting boundaries are used,

$$
\Phi=\partial_{r} \Phi=\Psi=\Theta=0 \quad \text { at } \quad r=r_{i}, r_{o} .
$$

The conduction state in nondimensional units is $T_{c}(r)=T_{0}+R a \eta / \sigma(1-\eta)^{2} r$.

The equations are discretized and integrated as described in [15] and references therein. The potentials and the temperature perturbation are expanded in spherical harmonics in the angular coordinates, and in the radial direction a collocation method on a Gauss-Lobatto mesh is used. The code is parallelized in the spectral and in the physical space by using OpenMP directives (see [14]). We use optimized libraries (FFTW3 [12]) for the FFTs in $\varphi$ and matrix-matrix products (dgemm GOTO [20]) for the Legendre transforms in $\theta$ when computing the nonlinear terms.

Copyright ( $\odot$ by SIAM. Unauthorized reproduction of this article is prohibited. 
For the time integration, high order implicit-explicit backward differentiation formulas (IMEX-BDF) $[15,16]$ are used. In the IMEX method we treat the nonlinear terms explicitly in order to avoid solving nonlinear equations at each time step. The Coriolis term is treated fully implicitly to allow larger time steps. The use of matrix-free Krylov methods (GMRES in our case) for the linear systems facilitates the implementation of a suitable order and time step size control.

3. Symmetry constrained convection at $R a \lesssim 2 R a_{c}$. In this section a study of the type of solutions of $(2.2 \mathrm{a})-(2.2 \mathrm{c})$ at the laminar regime is performed. The parameters are $\eta=0.35, \sigma=0.1$ (estimated for the Earth's outer core), $E=10^{-4}$, and $R a \lesssim 2 R a_{c}$. The critical parameters at the onset of convection, such as the critical Rayleigh number $R_{c}$, the critical precession frequency $\omega_{c}$, and the critical azimuthal wave number $m_{c}$, depending on $E$, were obtained in [17]. At $E=10^{-4}$ they are $R a_{c}=1.86 \times 10^{5},\left|\omega_{c}\right|=5.06 \times 10^{2}$, and $m_{c}=6$. According to [10] the first bifurcation, which breaks the axisymmetry of the conductive state, is a supercritical Hopf bifurcation giving rise to a wave, traveling in the azimuthal direction. With the preceding parameters, the preferred eigenfunctions are symmetric with respect to the equator, and they have negative precession frequencies $\omega_{c}$, namely, the drifting velocities $c=-\omega_{c} / m$ are positive, and the waves travel in the prograde direction. Moreover, they consist of quasi-geostrophic convective columns attached to the inner sphere and confined in a coaxial cylinder of radius $r_{c}$.

To obtain branches of periodic and quasi-periodic flows of fixed azimuthal wave number, the numerical solutions are computed by imposing an $m_{d}$-fold azimuthal symmetry. This is accomplished by retaining only the modes with wave number $m=k m_{d}, k \in \mathbb{Z}$, for a given $m_{d}$, in the truncated spherical harmonics expansion of the unknowns. The constraint on the symmetry allows us to find unstable flows. A perturbation without azimuthal symmetry $\left(m_{d}=1\right)$ is added to some solutions to check their stability. If the perturbation grows, and the original azimuthal symmetry is broken, then the solution is unstable.

The first solution of the $m=6$ branch, corresponding to $R a=2 \times 10^{5}$, is computed by starting from an initial condition with velocity $\mathbf{v}=\mathbf{0}$ and temperature

$$
T_{B}(r, \theta, \varphi)=\frac{r_{i} r_{o}}{r}-r_{i}+\frac{2 A}{\sqrt{2 \pi}}\left(1-x^{2}\right)^{3} P_{m}^{m}(\theta) \cos m \varphi,
$$

with $A=0.1, x=2 r-r_{i}-r_{o}, m=6$, and $P_{m}^{m}(\theta)=\sqrt{(2 m+1) ! ! / 2(2 m) ! !} \sin ^{m} \theta$ the normalized associated Legendre function of order and degree $m$. The solution tends, after an abrupt transient, to an azimuthal traveling wave of wave number $m=6$. At higher $R a$ the calculations are started from the solution computed at the previous lower $R a$. The resolution is $N_{r}=32$ and $L=27 m_{d}$, and it is increased from time to time in order to look into spatial discretization errors. A detailed numerical study of the effects of the truncation parameters at the same parameter values can be found in [18]. For instance, for values of $R a$ up to $10^{6}$ we obtained differences below $1 \%$ between the time-averaged physical properties when increasing the radial resolution from $N_{r}=32$ to $N_{r}=50$, and the spherical harmonic truncation parameter from $L=54$ to $L=84$. The initial transients are discarded until a stationary pattern is reached or until the time-averaged properties and the fundamental frequencies do not substantially change. To compute the other branches with $m=4,5,7$ we act in a similar way.

Copyright $\odot$ by SIAM. Unauthorized reproduction of this article is prohibited. 

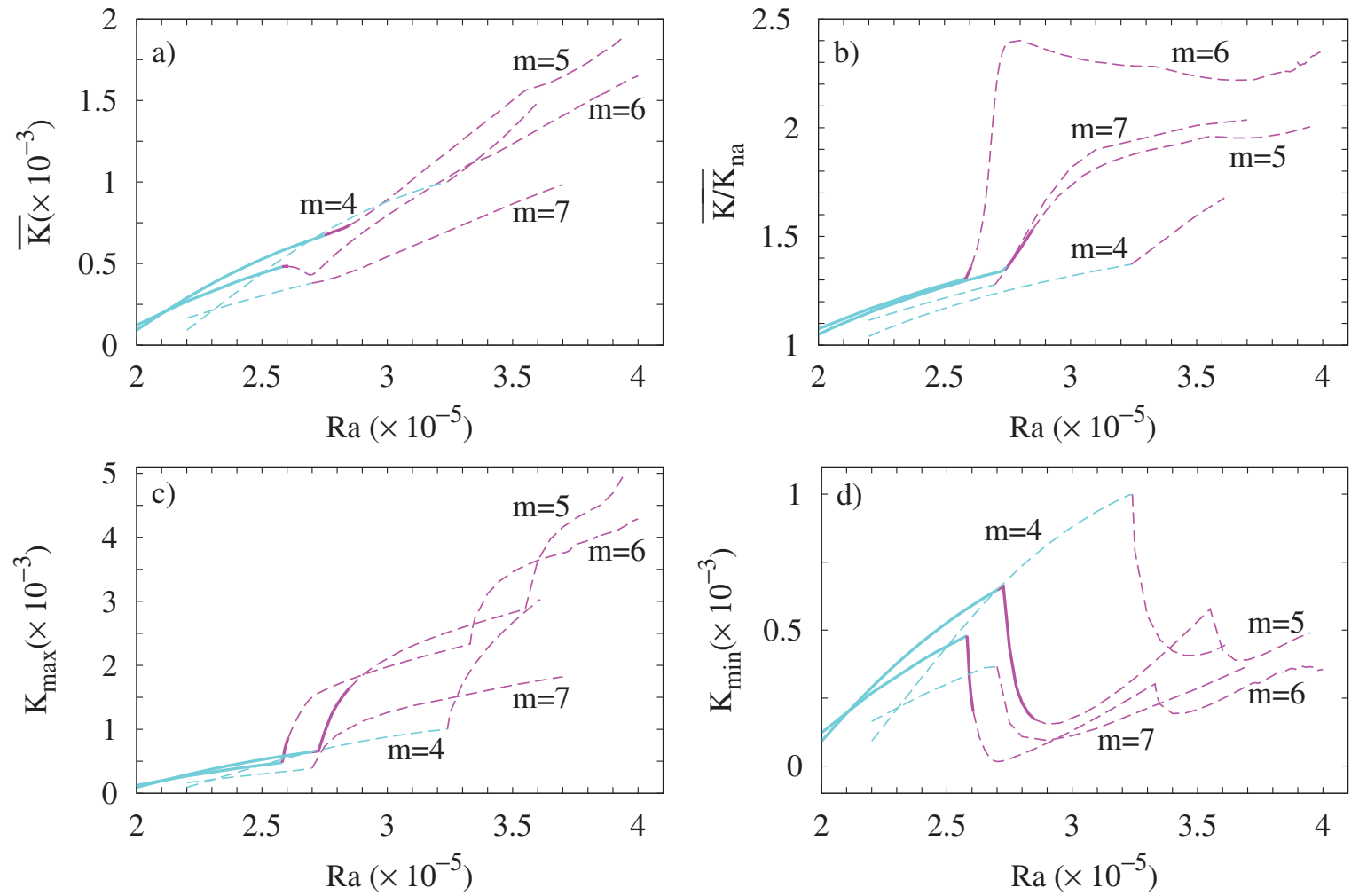

Figure 1. (a) Time average of the kinetic energy density, $K$, plotted versus the Rayleigh number, Ra, for solutions with azimuthal symmetry $m$. (b) Same as in (a) but for the ratio between the total and the nonaxisymmetric mean kinetic energy densities, $K / K_{n a}$. (c) and (d) The maximum and minimum of $K$, respectively, also plotted versus Ra. Traveling and modulated waves are represented in cyan and magenta, respectively. Stable and unstable solutions are indicated with solid and dashed lines, respectively.

For the sequence of solutions of each azimuthal wave number $m=4, \ldots, 7$ we will obtain some physical properties such as the time- and volume-averaged kinetic energy density, $\bar{K}$, where the volume-averaged kinetic energy density is $K=\frac{1}{2}\left\langle|\mathbf{v}|^{2}\right\rangle_{\mathcal{V}}$, i.e.,

$$
K=\frac{1}{\mathcal{V}} \int_{\mathcal{V}} \frac{1}{2}(\mathbf{v} \cdot \mathbf{v}) d v=\frac{3 \sqrt{2}}{4\left(r_{o}^{3}-r_{i}^{3}\right)} \int_{r_{i}}^{r_{o}} r^{2}\left[v^{2}\right]_{0}^{0}(r, t) d r .
$$

The previous volume integral can also be computed for either the axisymmetric, $K_{a}$, and the nonaxisymmetric, $K_{n a}$, kinetic energy densities defined by modifying the velocity field of (3.2). They are based, respectively, on the $m=0$ or the $m \neq 0$ modes of the spherical harmonic expansion of the potentials $\Psi$ and $\Phi$.

In Figures 1(a),(c),(d) the time average (mean from now on), maximum and minimum of the kinetic energy density, $K$, and time series are plotted, respectively, versus $R a$, for azimuthal wave numbers $m=4, \ldots, 7$. In Figure $1(\mathrm{~b})$ the time average of the ratio of the kinetic energy density over its nonaxisymmetric part, $\overline{K / K_{n a}}$, is also plotted versus $R a$ to quantify the importance of the nonaxisymmetric motions. Solid lines correspond to stable solutions, while dashed lines correspond to the unstable ones. Cyan is used for the branch

Copyright (C) by SIAM. Unauthorized reproduction of this article is prohibited. 
Table 1

The frequencies $f_{1}$ and $f_{2}$ of the time series of the temperature perturbation $\Theta$ at the point $(r, \varphi, \theta)=$ $\left(r_{i}+\left(r_{o}-r_{i}\right) / 7,0,3 \pi / 8\right)$ for solutions lying on the $m=6$ branch. The frequency $f_{2}$ corresponds to that of the volume-averaged physical properties such as $K, K_{n a}$, etc.

\begin{tabular}{ccccc}
\hline$R a$ & $R a / R a_{c}$ & $f_{1}$ & $f_{2}$ & Period doubling \\
\hline $2.50 \times 10^{5}$ & 1.347 & 61.749 & & \\
$2.55 \times 10^{5}$ & 1.374 & 60.634 & & \\
$2.58 \times 10^{5}$ & 1.390 & 59.973 & 27.356 & \\
$2.63 \times 10^{5}$ & 1.417 & 61.181 & 25.141 & \\
$3.00 \times 10^{5}$ & 1.616 & 67.788 & 33.933 & \\
$3.33 \times 10^{5}$ & 1.794 & 63.826 & 44.371 & \\
\hline $3.34 \times 10^{5}$ & 1.800 & 63.661 & 22.176 & \\
$3.71 \times 10^{5}$ & 2.000 & 56.757 & 24.795 & first \\
$3.73 \times 10^{5}$ & 2.010 & 56.444 & 25.007 & \\
\hline $3.74 \times 10^{5}$ & 2.015 & 56.328 & 12.576 & \\
$3.80 \times 10^{5}$ & 2.047 & 55.657 & 12.985 & second \\
$3.82 \times 10^{5}$ & 2.058 & 55.447 & 13.117 & \\
\hline $3.83 \times 10^{5}$ & 2.064 & 55.323 & 6.5858 & \\
$3.84 \times 10^{5}$ & 2.069 & 55.199 & 6.6132 & third \\
$3.85 \times 10^{5}$ & 2.074 & 55.091 & 6.6431 & \\
\hline
\end{tabular}

of periodic solutions corresponding to traveling waves whose columnar pattern drifts in the azimuthal direction without change in the volume-averaged physical properties. Magenta is used for the quasi-periodic solutions, which are modulated waves. In this case, in addition to the azimuthal drift, the columnar pattern and its volume-averaged physical properties are modulated by a second frequency.

At the appearance of the modulated waves, $\bar{K}$ decreases for $m=5,6,7$ when increasing $R a$ (see Figure 1(a)) because of the larger decrease of the minimum of $K$ when compared with the increase of the maximum (see Figures $1(\mathrm{c})-(\mathrm{d})$ ). At the same time the ratio $\overline{K / K_{n a}}$ increases sharply, meaning that the axisymmetric component of the flow is enhanced by the onset of the modulated waves (see Figure 1(b)). As will be shown in the next section, this behavior can be reproduced by a simple model of five degrees of freedom. In the particular case of the unstable quasi-periodic solutions lying in the $m=5,6$ branches, we have found a sequence of period doubling bifurcations concerning the modulating frequency. These period doublings can be identified in the cusps of the $m=6$ curve in Figure 1(d) for $R a>3.33 \times 10^{5}$. Without symmetry assumptions and when the Rayleigh number is increased from that of the onset of convection, we first obtain the $m=6$ periodic orbits (traveling waves) followed by a small parameter region of two- and three-frequency solutions. If the Rayleigh number is further increased, the solution jumps to the $m=5$ periodic orbits branch. By further increasing the Rayleigh number, an also small stability region of two- and three-frequency solutions is traversed. Beyond these regimes, heteroclinic cycles connecting the unstable $m=4$ traveling waves and the unstable $m=5$ modulated waves, as described in [34], are found.

In Table 1 the frequencies of the time series of the temperature perturbation $\Theta$ at the point $(r, \varphi, \theta)=\left(r_{i}+\left(r_{o}-r_{i}\right) / 7,0,3 \pi / 8\right)$ for some solutions corresponding to the $m=6$

Copyright $\odot$ by SIAM. Unauthorized reproduction of this article is prohibited. 
branch are shown. They are computed by using the Laskar algorithm of fundamental frequencies [23], which allows an accurate determination of the frequencies with larger amplitude of the spectrum. The time series have at least $10^{5}$ points corresponding to a time integration of 10 viscous time units. The frequency denoted by $f_{1}$ corresponds to that of the azimuthal drift, while the second frequency $f_{2}$ is that of the modulation of the volume-averaged physical properties.

The frequency of the $m=6$ traveling waves decreases monotonically, because of the action of the Reynolds stress due to the spiraling of the traveling waves [36]. In addition, the convection remains confined in a cylinder whose critical radius $r_{c}$ becomes larger, and the heat transfer through the outer boundary increases. The increase of the spiraling nature of the flow also favors the zonal circulation, which increases monotonically with the Rayleigh number, as can be seen in the ratio $\overline{K / K_{n a}}$ in Figure 1(b). Notice that in the latter figure the axisymmetric flow of the traveling waves carries only less than $20 \%$ of the total kinetic energy density. This is a characteristic of the non-slip boundary conditions (see $[8,18]$ and references therein). At approximately $R a_{1}=2.59 \times 10^{5}$, the $m=6$ traveling wave starts to oscillate with an additional frequency $f_{2}$. As $R a$ is increased, $f_{2}$ decreases, reaching a relative minimum at $R a \approx 2.7 \times 10^{5}$, and increases again as for $\bar{K}$ in Figure 1 (a). In contrast, $f_{1}$ increases and at approximately $R a \approx 2.8 \times 10^{5}$ starts to decrease again. As mentioned before, at $R a>3.33 \times 10^{5}$ the second frequency halves due to the period doubling bifurcation, and successive period doublings occur with a further increase of $R a$. Notice that the ratio of the differences between the three Rayleigh numbers at which the period doubles is already 4.44 , roughly the Feigenbaum constant. In addition, the limit of the period-doubling cascade would be approximately $R a=3.85 \times 10^{5}$. We have found, as expected, chaotic attractors above this limit. It must be stated that these solutions are attracting only when restricted to an invariant manifold of fixed azimuthal symmetry. Without this constraint these objects are of saddle type, eventually giving rise to transient chaotic behavior.

Aside from the analysis of the frequency spectrum, Poincaré sections can also be used to identify two frequency solutions (invariant tori). This is shown in Figure 2, where the Poincaré section at $\Theta\left(\left(r_{o}+r_{i}\right) / 2,0,3 \pi / 8\right)=0$ of the azimuthal component of the velocity field $\mathbf{v}_{\varphi}\left(r_{i}+\left(r_{o}-r_{i}\right) / 7,0,3 \pi / 8\right)$ is plotted versus $\Theta$ at the same point. Figure 2(a) corresponds to stable solutions of the $m=6$ branch, while Figure 2(b) corresponds to stable solutions of the $m=5$ branch. The Rayleigh number increases from inner to outer curves. We have identified a resonant solution (frequency locking) at $R a=2.59929964 \times 10^{5}$ (the set of nine points in Figure $2(\mathrm{a})$ ) with $f_{2} / f_{1}=4 / 9$, and a quasi-resonant solution at $R a=2.78302 \times 10^{5}$ (the set of nine segments in Figure $2(\mathrm{~b}))$ also with $f_{2} / f_{1} \approx 4 / 9$.

Figures 3(a)-(c) show the Poincaré sections corresponding to solutions at $R a=3.71 \times$ $10^{5}, 3.8 \times 10^{5}, 3.84 \times 10^{5}$ with the first, second, and third period doublings of the modulating frequency, respectively, lying on the $m=6$ branch. In Figure 3(d) the Poincare section of a temporal chaotic solution at $R a=3.855 \times 10^{5}$ is also shown. After the appearance of chaos, no relaminarized solutions were found by following the same sequence of bifurcations. The obtention of enough points for these sections requires time integrations of about 100 viscous time units (i.e., time series with $10^{6}$ points).

The time series of the axisymmetric (red solid line) and nonaxisymmetric (black dashed line) kinetic energy density for the four solutions included in Figure 3 are shown in Figure 4.

Copyright (c) by SIAM. Unauthorized reproduction of this article is prohibited. 

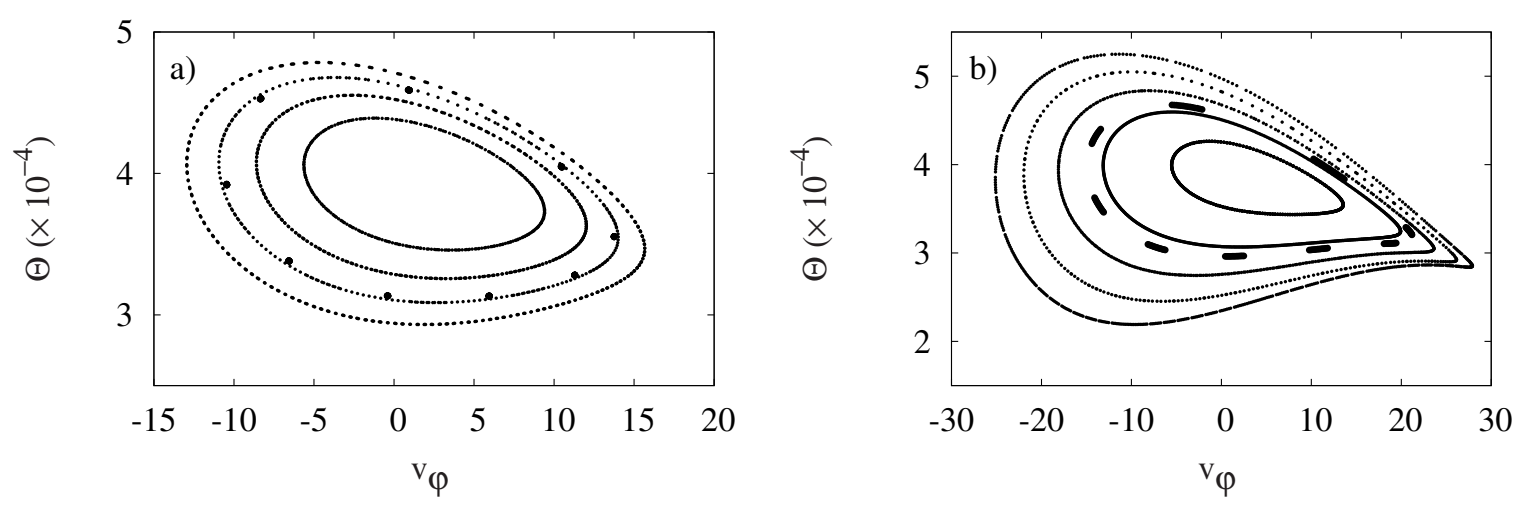

Figure 2. (a) Poincaré section at $\Theta\left(\left(r_{o}+r_{i}\right) / 2,0,3 \pi / 8\right)=0$ of the azimuthal component of the velocity field $\mathbf{v}_{\varphi}\left(r_{i}+\left(r_{o}-r_{i}\right) / 7,0,3 \pi / 8\right)$, plotted versus $\Theta$ at the same point. The solutions correspond to stable invariant tori with $m=6$. The Rayleigh numbers, from inner to outer curves, are $R a=2.59 \times 10^{5}, 2.595 \times 10^{5}, 2.59929964 \times$ $10^{5}, 2.6 \times 10^{5}, 2.605 \times 10^{5}$. The set of nine points corresponds to the resonant solution at $R a=2.59929964 \times 10^{5}$. (b) As in (a) but for solutions corresponding to stable invariant tori with $m=5$. The Rayleigh numbers, from inner to outer curves, are $R a=2.75 \times 10^{5}, 2.775 \times 10^{5}, 2.78302 \times 10^{5}, 2.8 \times 10^{5}, 2.825 \times 10^{5}, 2.85 \times 10^{5}$.

In these figures the period doubling can be clearly identified. The time average of the axisymmetric kinetic energy density is always smaller than that of the nonaxisymmetric, and the positions of the relative extrema are shifted. At first sight the time series of Figure 4(d) does not seem to correspond to a temporal chaotic solution; however, its Poincaré section and frequency spectrum indicate clearly the chaotic behavior.

4. Flow patterns of quasi-periodic convection. In this section the time evolution of some physical properties and flow patterns will be shown to visualize the behavior of the modulated waves after the period doubling bifurcation.

Figure 5(a) shows the time series of the kinetic energy density of the $m=6$ unstable modulated wave with period doubled at $R a=3.71 \times 10^{5}$. The radial profile of the azimuthally averaged azimuthal velocity (mean zonal flow), $\left\langle v_{\varphi}\right\rangle$, on the equatorial plane for the time instants plotted with (*) in Figure 5(a) are displayed in Figure 5(b) (see movie M100729_01.gif [local/web 7.35MB]). Figure 5(c) (see movie M100729_02.gif [local/web 9.89MB]) contains curves that are equivalent to those of (b) but are for the radial profiles of the axial vorticity $\left\langle w_{z}^{2}\right\rangle^{1 / 2}$, which quantify the location and radial length scale of the convective cells. Near the inner boundary the mean zonal flow is negative (retrograde), and positive (prograde) in the middle of the shell, reaching its largest values at the time instant $t_{2}$ (green curve). The value of $\left\langle w_{z}^{2}\right\rangle^{1 / 2}$ is maximum near $r \approx 0.9$, meaning that large-scale cells are located close to the radial point where $\left\langle v_{\varphi}\right\rangle$ is nearly stagnant and with nearly zero modulus. When the time evolves, the maximum of $\left\langle w_{z}^{2}\right\rangle^{1 / 2}$ decreases while moving to the outer boundary, and then increases while moving to the inner. Notice the large value of $\left\langle w_{z}^{2}\right\rangle^{1 / 2}$ at the inner boundary due to the existence of a thin Ekman layer. It can be understood by taking into account that the axial vorticity at the equator is $w_{z}=-w_{\theta}$. Approximating the latter in terms of the velocity field close to the non-slip boundaries $r=r_{i}, r_{o}$, we obtain $w_{\theta} \approx-\partial_{r}\left(v_{\varphi}\right)$. This radial derivative can be estimated from Figure 5(b). It is clear that the large value of $\left\langle w_{z}^{2}\right\rangle^{1 / 2}$

Copyright (c) by SIAM. Unauthorized reproduction of this article is prohibited. 

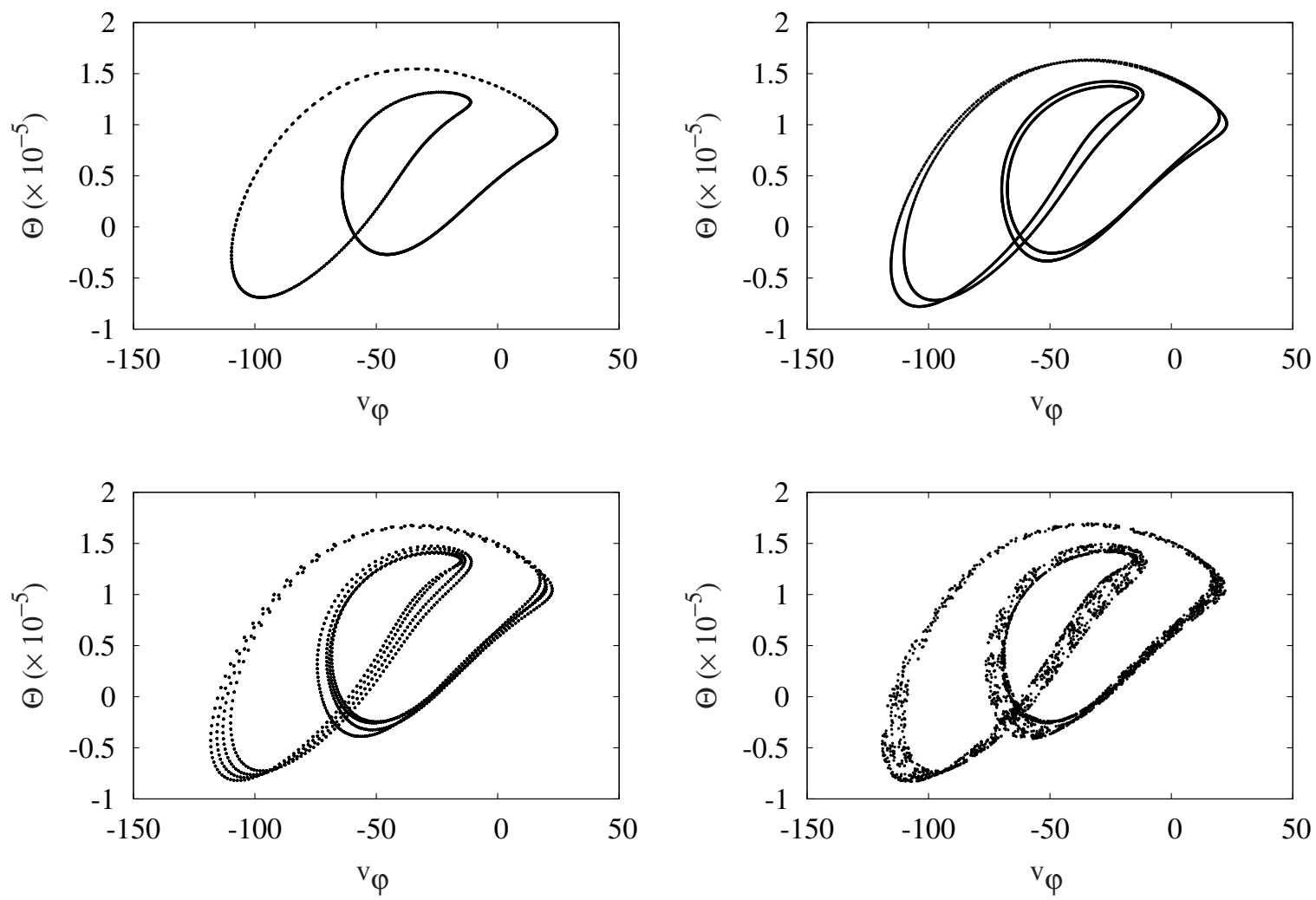

Figure 3. Poincaré section at $\Theta\left(\left(r_{o}+r_{i}\right) / 2,0,3 \pi / 8\right)=0$ of the azimuthal component of the velocity field $\mathbf{v}_{\varphi}\left(r_{i}+\left(r_{o}-r_{i}\right) / 7,0,3 \pi / 8\right)$, plotted versus $\Theta$ at the same point. The Rayleigh numbers, from top to bottom and from left to right, are $3.71 \times 10^{5}, 3.8 \times 10^{5}, 3.84 \times 10^{5}, 3.855 \times 10^{5}$. First, second, and third doublings of the period of the modulation and a chaotic solution are shown in the sequence of plots.

shown in Figure 5(c) at the inner boundary corresponds to the large radial derivative of $v_{\varphi}$ in Figure 5(b), in contrast to what happens at the outer boundary, where both values are smaller.

The flow patterns of the unstable $m=6$ modulated wave bifurcated at $R a=3.71 \times 10^{5}$ are shown in Figures 6 and 7. Figure 6 shows, from top to bottom, a sequence of five snapshots taken at the even time instants $\left(t=t_{2}, t_{4}, t_{6}, t_{8}, t_{10}\right)$ shown in Figure 5 . Three projections of the axial vorticity are displayed in the plots in columns 1-3 (see movie M100729_03.gif [local/web 7.69MB]). The radius of the spherical surfaces is $r=r_{o}$, although they are represented with the same size as the other sections. The radius corresponds approximately to the location where the columns of vorticity reach their maximum. The projections in the middle column of each group of projections are taken on the equatorial plane, and the projections in the right column of each group are taken on a meridional section which rotates with the drifting frequency of the modulated wave to facilitate recognizing the ratio of the modulation to the drift frequencies. The scale of colors is the same for all of the contour plots corresponding to each time instant, with blue meaning negative axial vorticity. The same projections are taken for the azimuthal

Copyright (C) by SIAM. Unauthorized reproduction of this article is prohibited. 

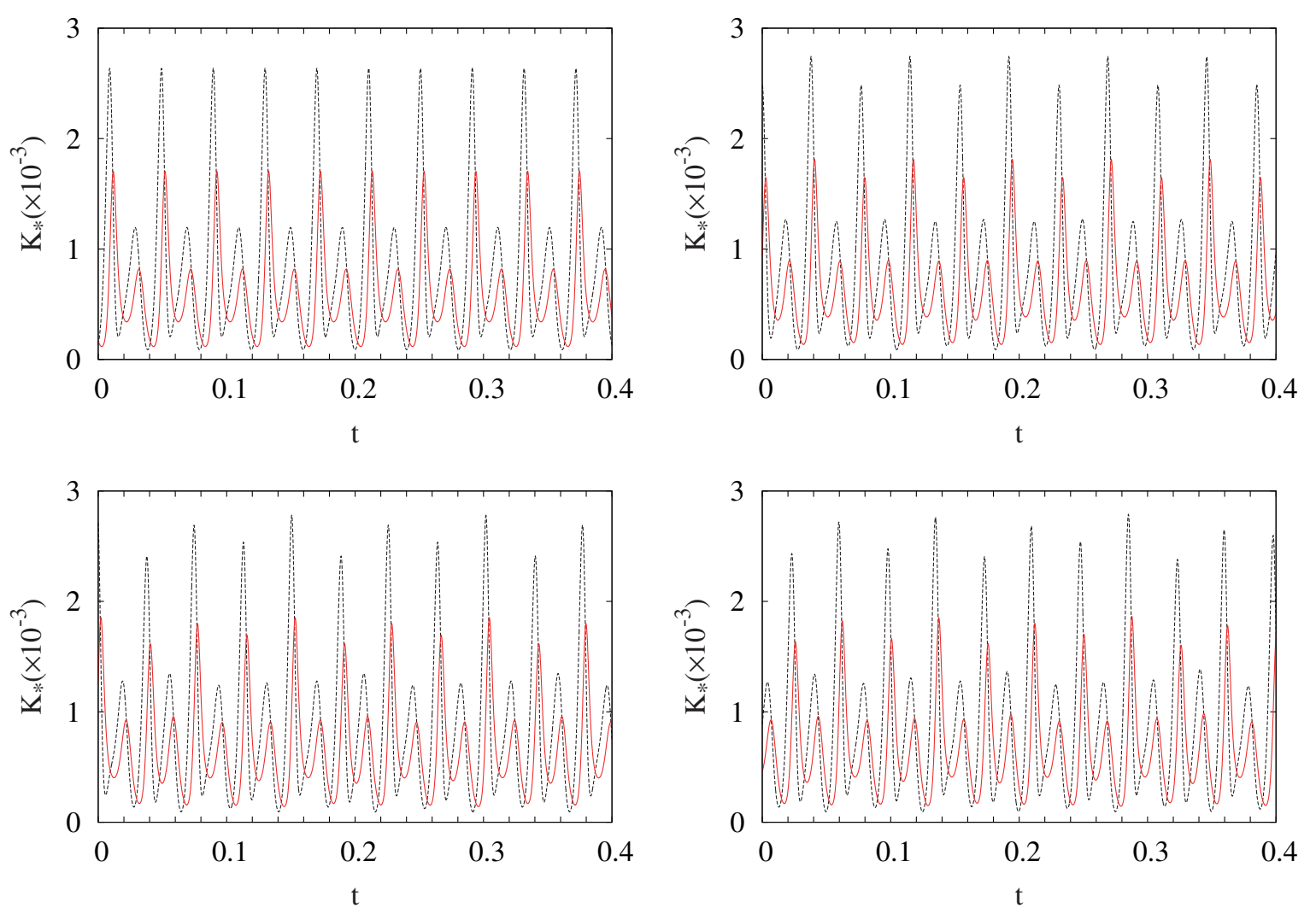

Figure 4. Time series of the axisymmetric (red solid line) and nonaxisymmetric (black dashed line) kinetic energy density. The Rayleigh numbers, from top to bottom and from left to right, are $R a=3.71 \times 10^{5}, 3.8 \times$ $10^{5}, 3.84 \times 10^{5}, 3.855 \times 10^{5}$.

velocity (plots in columns 4-6 and movie M100729_04.gif [local/web 9.29MB]), but with the spherical projections taken close to the outer boundary at $r=r_{i}+0.975 d$, where it reaches its maximum at high latitudes. In these contour plots the time intervals are selected to follow the period of the kinetic energy density time series of Figure 5(a). In the same manner, Figure 7 shows the contour plots of the temperature perturbation (see movie M100729_05.gif [local/web $642 \mathrm{~KB}]$ ), with the spherical projection taken at $r=r_{i}+0.325 d$ close to its maximum, and the contour plots of the kinetic energy density (see movie M100729_06.gif [local/web 745KB]) projected onto a spherical surface of radius $r=r_{i}+0.975 d$ with a polar point of view.

The meridional sections show clearly that the $z$-dependence of the flow is weak, and that it is symmetric with respect to the equatorial plane. In addition, convection inside the tangent cylinder is nearly absent but extends to most of the remainder of the shell. The equatorial sections of the azimuthal velocity and the kinetic energy density display a double-layered pattern with spiraling cells. The spiral vortices shown in the equatorial section of the axial vorticity develop close to the shear region between the double-layered structure and reach their maxima close to the outer surface. The boundary of the two regions is located around $r \approx r_{i}+d / 3$. 

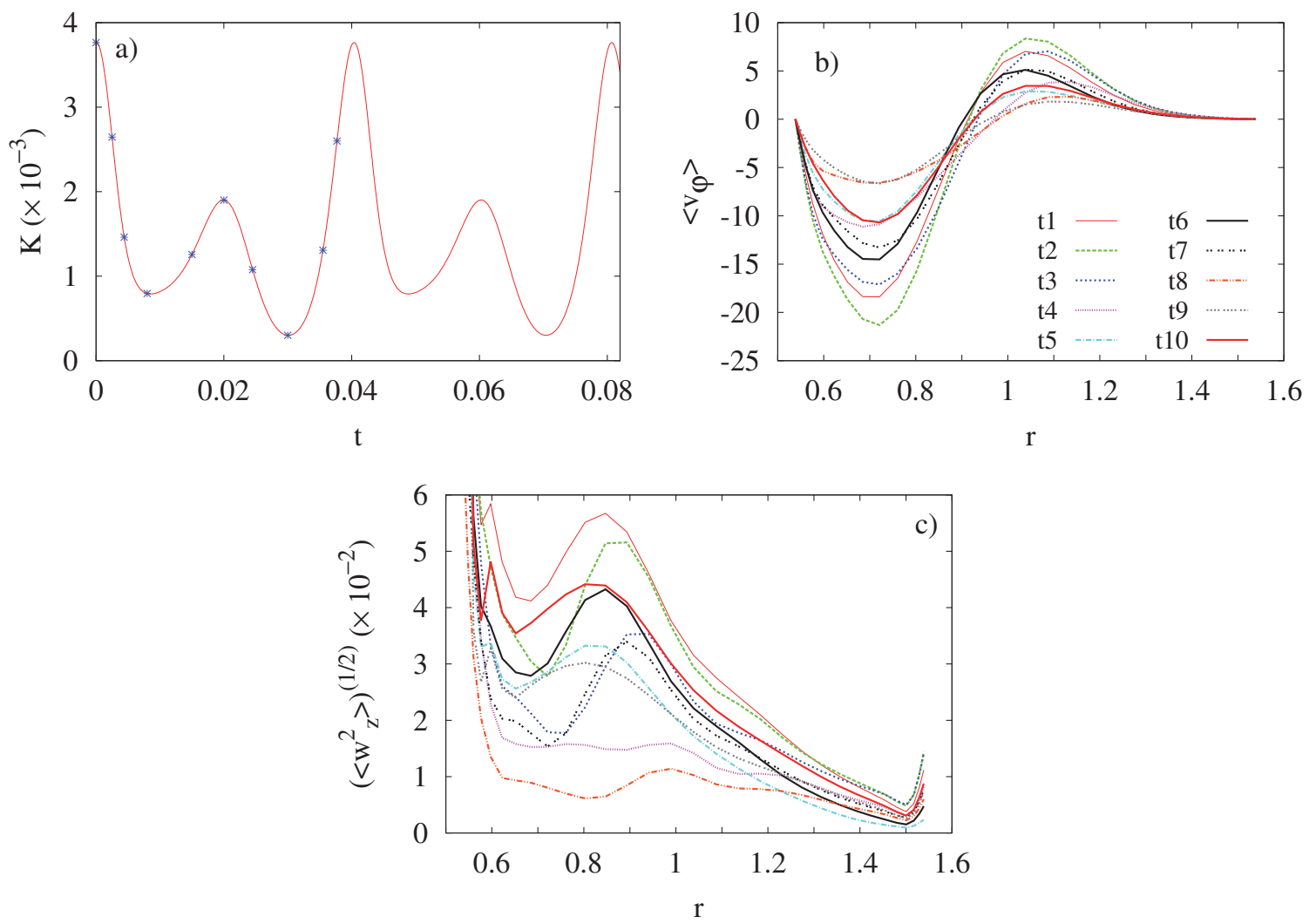

Figure 5. (a) Time series of the kinetic energy density, $K$. The symbols (*) indicate the time instants at which the radial profiles of (b), (c) are taken. (b) Radial profile of the mean zonal flow, $\left\langle v_{\varphi}\right\rangle$, on the equatorial plane for the time instants indicated in (a). (c) Same as in (b) but for the axial vorticity $\left\langle w_{z}^{2}\right\rangle^{1 / 2}$. The solution corresponds to an unstable $m=6$ torus with period doubled at $R a=3.71 \times 10^{5}$.

5. A simple model for weakly nonlinear quasi-periodic convection. In [40] the onset of small-Prandtl-number convection was described at leading order as the superposition of a small number of quasi-geostrophic-inertial modes (the number depending on $\sigma$ ) of same azimuthal wave number and different radial configuration, modified by an oscillatory boundary layer flow. In this section it will be shown that it is also possible to identify the dynamics of the modulated waves with the nonlinear interaction of two convective modes $\psi, \phi \in \mathbb{C}$ with the same azimuthal symmetry and different radial structure and a mode $u \in \mathbb{R}$ representing the zonal flow. The mode $\psi$ will be associated with the rigid rotation of the traveling wave, and the mode $\phi$ with the modulation of the modulated waves.

Our model is purely empirical, described by an ODE system for $\psi, \phi, u$, depending on parameters $\mu=\mu_{r}+i \mu_{i} \in \mathbb{C}$ and $\lambda, \alpha, \beta>0 \in \mathbb{R}$, with a bifurcation diagram depending on $\mu_{r}$ of behavior similar to that obtained with the simulation of the full Boussinesq NavierStokes equations restricted to manifolds of fixed azimuthal symmetry: a fixed point (the conductive state), traveling waves, modulated waves, modulated waves with period doubling, and a chaotic solution.

Copyright (C) by SIAM. Unauthorized reproduction of this article is prohibited. 

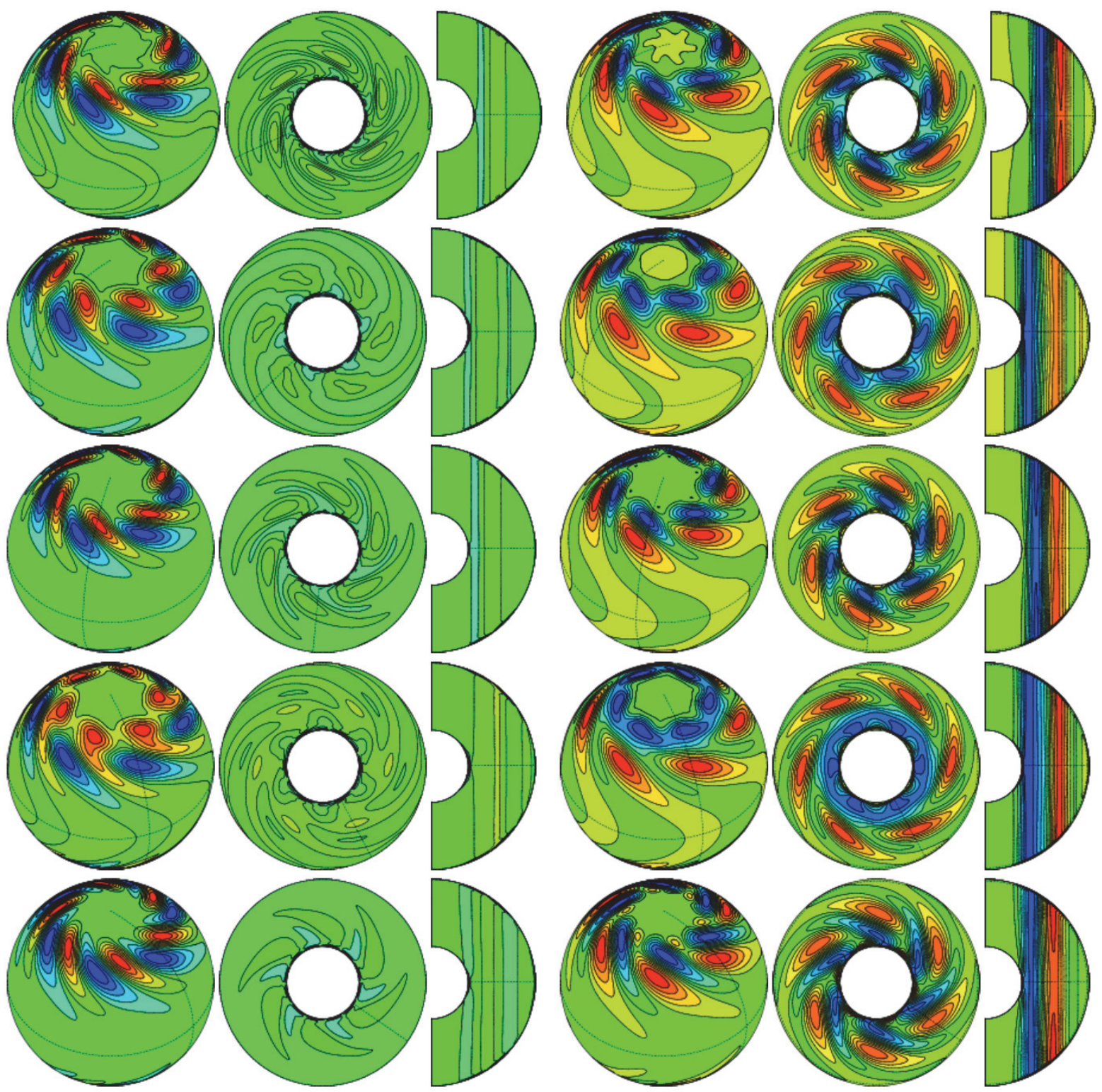

Figure 6. The first three columns are contour plots of the axial vorticity for the five even time instants marked with (*) in Figure 5(a). The first column shows projections on a sphere, the second on the equatorial plane, and the last on a meridional section. Columns 4-6 contain the same projections but for the azimuthal velocity. As in the preceding figure, the solution corresponds to an unstable $m=6$ torus.

Copyright (C) by SIAM. Unauthorized reproduction of this article is prohibited. 

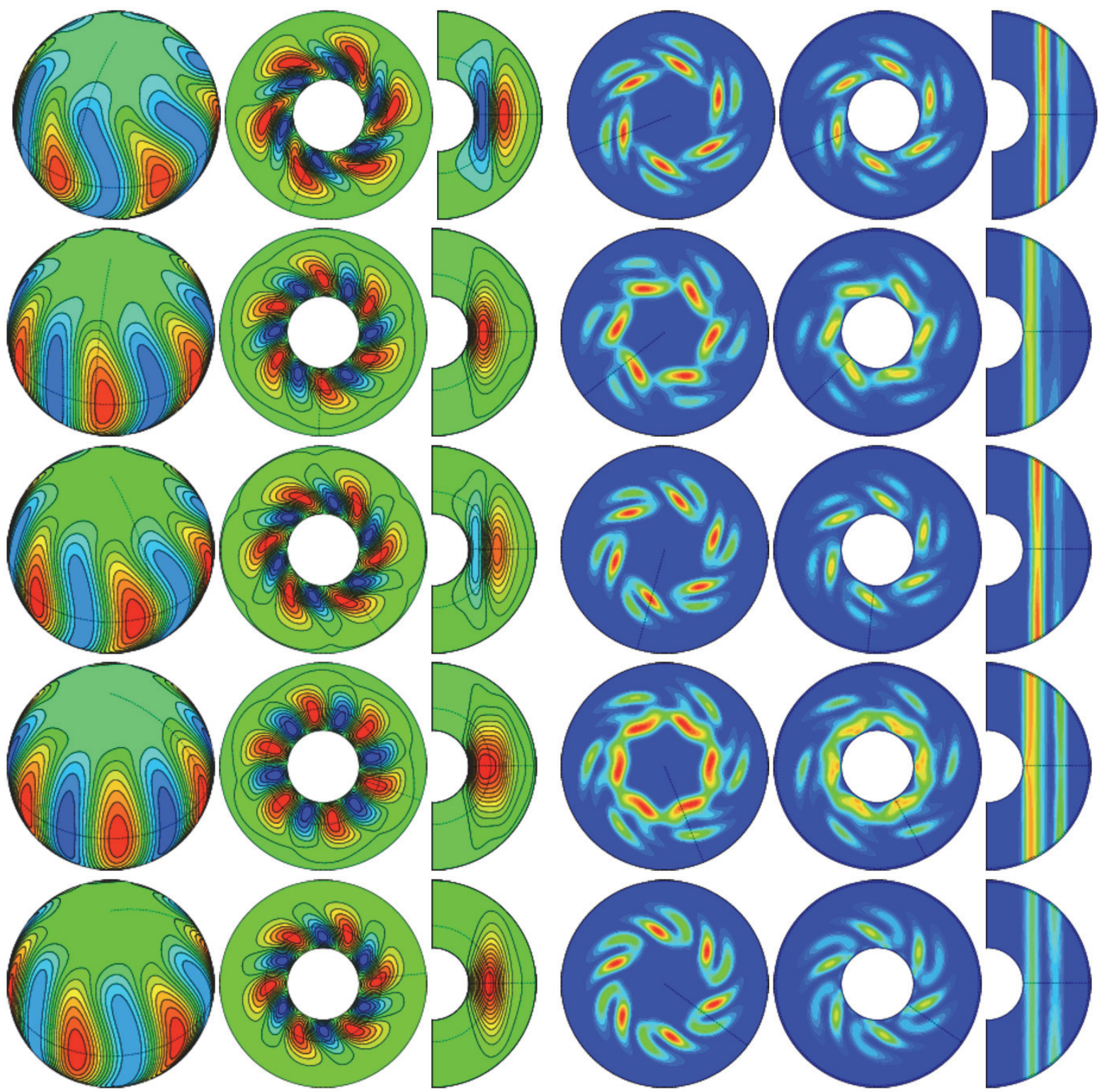

Figure 7. Same as in Figure 6 for the temperature perturbation in columns 1-3, and the kinetic energy density in columns $4-6$.

As $\psi$ and $\phi$ represent modes with the same azimuthal symmetry, the equations must be invariant under the transformations $\tilde{\psi} \rightarrow \psi e^{i \gamma}, \tilde{\phi} \rightarrow \phi e^{i \gamma}$, and $\tilde{u} \rightarrow u$. We retain the following second order nonlinear interactions:

$$
\begin{aligned}
& \dot{\psi}=\mu \psi-\alpha u \psi, \\
& \dot{\phi}=-\phi-u \phi-|\phi|^{2} \psi, \\
& \dot{u}=-\beta u+\lambda|\psi|^{2}+|\phi|^{2} .
\end{aligned}
$$

Copyright (C) by SIAM. Unauthorized reproduction of this article is prohibited. 

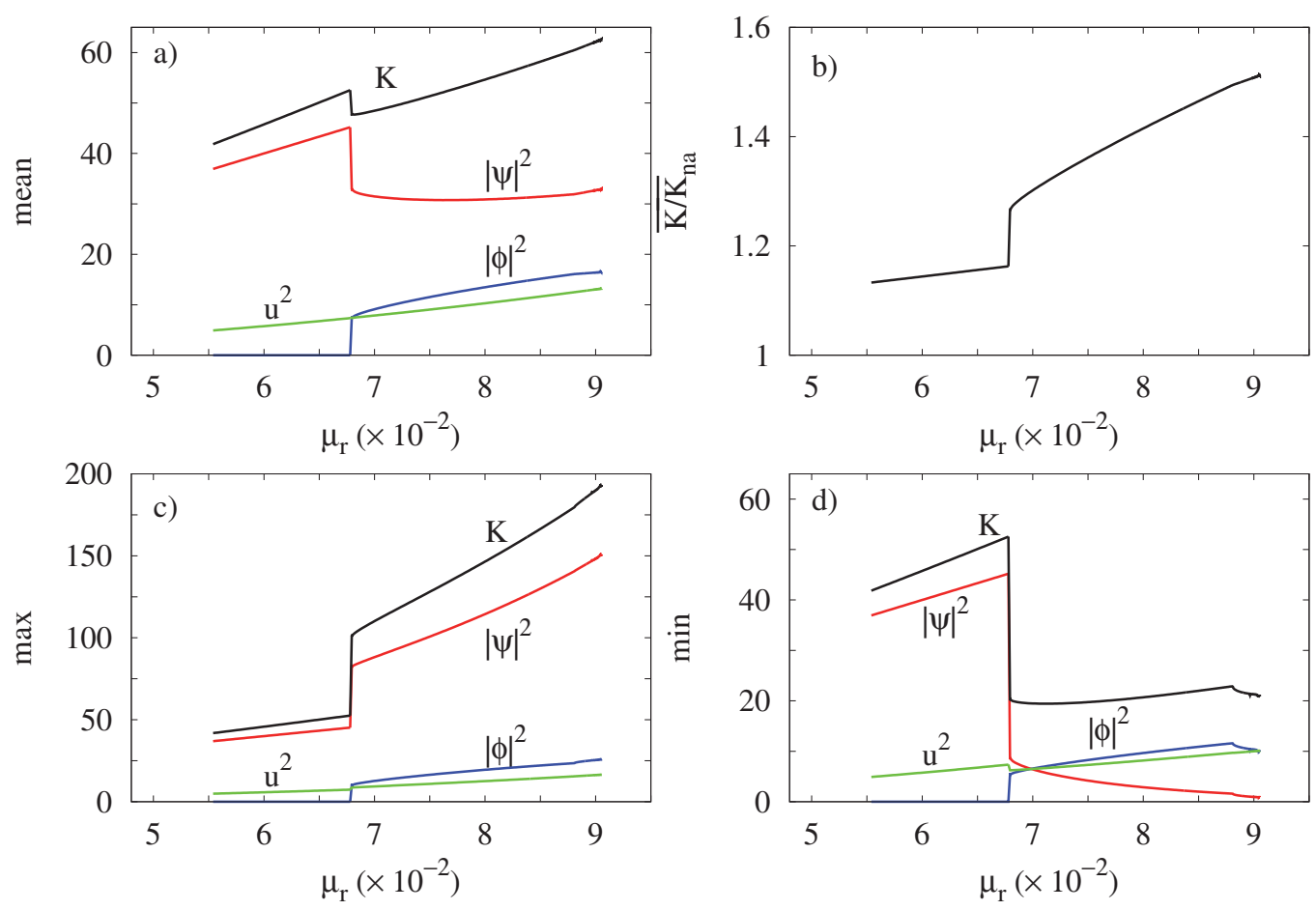

Figure 8. (a) Time averages of $|\psi|^{2},|\phi|^{2}, u^{2}$, and $K=|\psi|^{2}+|\phi|^{2}+u^{2}$ plotted versus $\mu_{r}$ for solutions of the low-dimensional model. (c) Same as in (a) but for the maximum values. (d) Same as in (a) but for the minimum values. (b) Time average of the ratio between the total and the nonaxisymmetric kinetic energy densities, $K / K_{n a}$, plotted versus $\mu_{r}$. The parameters are $\alpha=250, \beta=10, \lambda=0.6$, and $\mu_{i}=100$.

This equation has a fixed point $\psi=\phi=u=0$ that undergoes a Hopf bifurcation when $\mu_{r}=0$. The solution $\psi=\left(\beta \mu_{r} / \alpha \lambda\right)^{1 / 2} e^{i\left(\mu_{i} t+c\right)}, \phi=0$, and $u=\mu_{r} / \alpha$ is stable and will represent a traveling wave (a periodic orbit). In addition, $\phi=0$ is invariant, and $\phi$ is enforced only by the term $-|\phi|^{2} \psi$. With this term the equations for the modulus and the phase of $\phi$ are coupled with the modulus and phase of $\psi$, giving rise to the modulated waves (twofrequency solutions). Notice that the term $-u \phi$ should have the minus sign because otherwise the equations admit a solution with $\psi=0$ and $u=|\phi|^{2} / \beta \neq 0$ (the mode which gives the modulation can survive without convection). We have obtained modulated waves for the following range of parameters:

- $\alpha=250, \beta=10, \lambda \in[0.6,1.4], \mu_{r}>680$, and $\mu_{i}=100$.

- $\alpha=1000, \beta=10, \lambda=0.0625, \mu_{r}>700$, and $\mu_{i}=100$.

The solutions are obtained numerically using a variable order solver based on the numerical differentiation formulas, with relative and absolute tolerances of $10^{-7}$ and $10^{-10}$, respectively.

In Figure 8(a) the time averages of $|\psi|^{2},|\phi|^{2}, u^{2}$, and $K=|\psi|^{2}+|\phi|^{2}+u^{2}$ are plotted versus $\mu_{r}$. The maximum and minimum of the time series are shown in Figures 8(c) and $8(\mathrm{~d})$, respectively. The time average of the ratio between $K$ and the nonaxisymmetric part $K_{n a}=|\psi|^{2}+|\phi|^{2}$ is shown in Figure 8(b). The parameters of (5.1)-(5.3) are $\alpha=250, \beta=10$, $\lambda=0.6$, and $\mu_{i}=100$. Notice that these figures are very similar to Figure 1, obtained with 

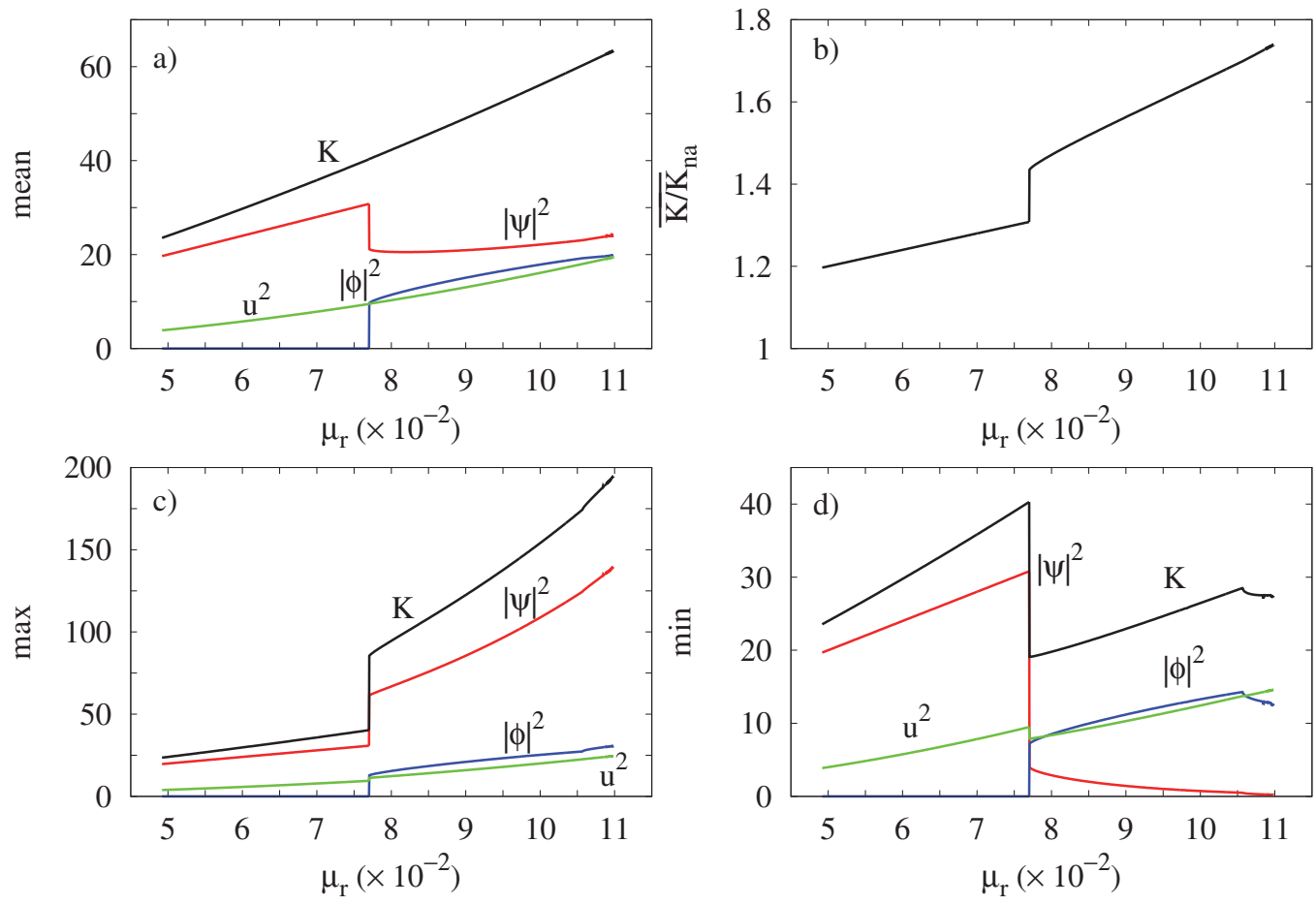

Figure 9. (a)-(d) Same as in Figure 8 but for $\lambda=1$.

the simulation of the full Boussinesq Navier-Stokes equations. They display the decrease of $\bar{K}$ when the traveling waves bifurcate to modulated waves together with an abrupt increase of the ratio $\overline{K / K_{n a}}$. In Figure $8(\mathrm{~d})$ the period doubling bifurcation can be identified by the cusp of $\min K$ shown at the end of the curve.

Figures 9 and 10 correspond to the bifurcation diagrams obtained by changing only the parameter $\lambda$ with respect to that of Figure 8 . We set $\lambda=1$ for Figure 9 , and $\lambda=1.4$ for Figure 10. The effect of changing $\lambda$ is to change the decrease of $\bar{K}$ (and also the increase of $\overline{K / K_{n a}}$ ) after the bifurcation of the traveling waves. For $\lambda=1$ there is no decrease (very similar to what happens on the $m=4$ branch of Figure 1), while for $\lambda=1.4$ there is even an increase.

The time series of the axisymmetric (red solid line) and nonaxisymmetric (black dashed line) kinetic energy densities for four solutions lying on the right part of Figure 8 at $\mu_{r}=900$, 904, 905.43, and 905.55 are shown in Figure 11. In these figures the period doubling can not be clearly identified. As happened for the full Boussinesq Navier-Stokes equations, the time average of the axisymmetric kinetic energy density is always smaller than that of the nonaxisymmetric, and the positions of the relative extrema are shifted.

To depict clearly the period doubling bifurcations of the modulated waves, some Poincaré sections at $\Re(\psi)=0$ of $\Im(\phi)$ plotted versus $\Re(\phi)$ are shown in Figure 12. Two modulated waves at $\mu_{r}=679.423$ and 750 together with a modulated wave after the first period doubling at $\mu_{r}=900$ are shown in Figure 12(a). The Poincaré section of the solution at $\mu_{r}=900$ develops a loop which indicates that the period is doubled. Figure 12(b) corresponds to an

Copyright (C) by SIAM. Unauthorized reproduction of this article is prohibited. 

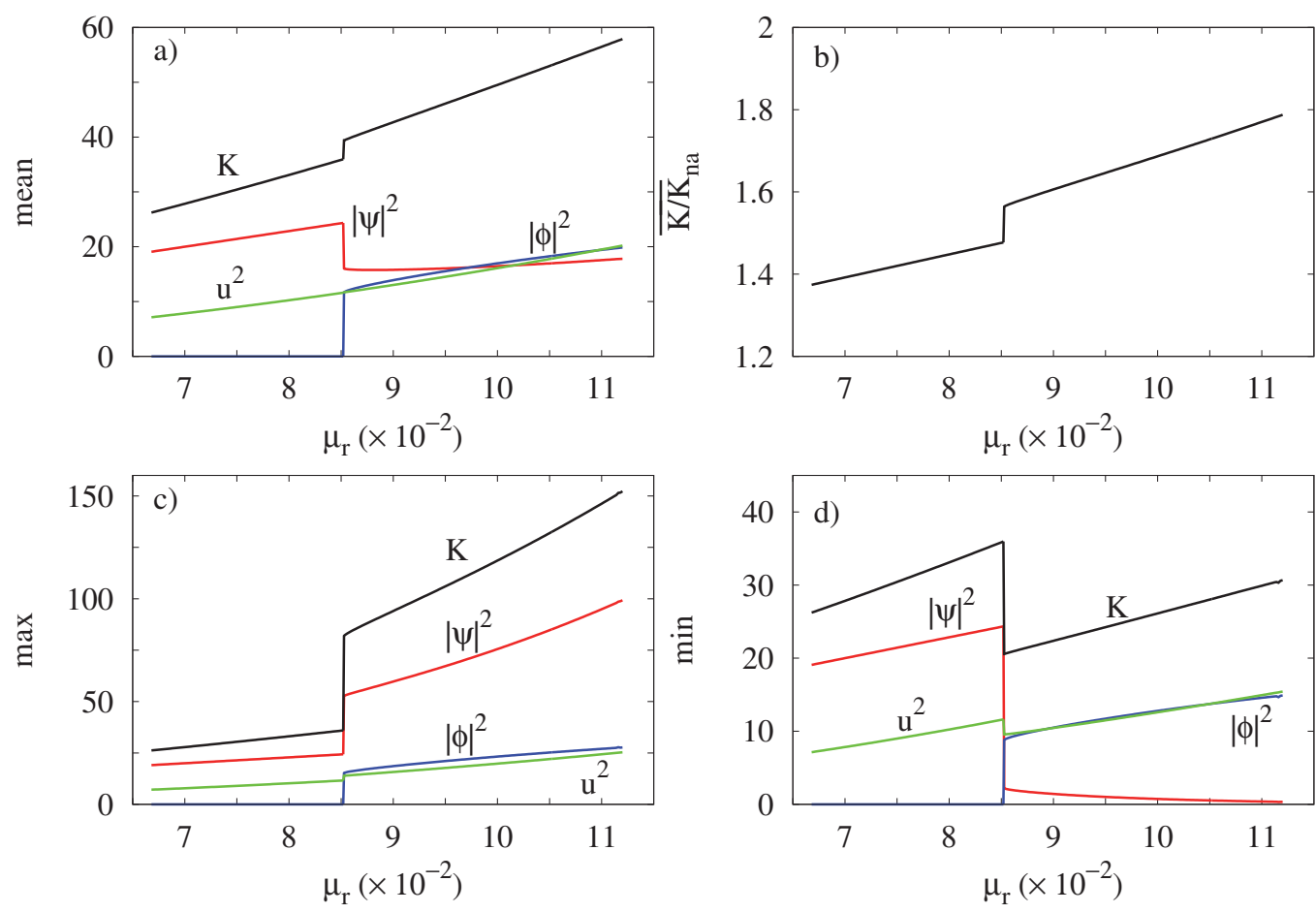

Figure 10. (a)-(d) Same as in Figure 8 but for $\lambda=1.4$.

amplification of the square box displayed in Figure 12(a). It also shows two solutions at $\mu_{r}=904$ and 905.43 corresponding to the second and third period doublings in green and red, respectively. In Figure 12(c) a solution with a fourth period doubling at $\mu_{r}=905.52$ is shown, and finally Figure 12(d) corresponds to the Poincaré section of the chaotic solution at $\mu_{r}=905.55$.

6. Summary. The regime of oscillatory Boussinesq thermal convection in fast rotating spherical shells is investigated carefully in this paper. The model and the parameters have been set as similar as numerically possible to those of the Earth's outer liquid core and previous experimental studies. Non-slip boundary conditions were poorly considered in previous numerical studies due to the numerical effort needed to solve the Ekman layers. If the Prandtl number decreases, for a fixed low Ekman number, the drifting frequency increases with the corresponding decreasing of the width of the Ekman layers. Moreover, low Prandtl numbers facilitate the appearance of oscillatory and chaotic convection at a weakly supercritical Rayleigh number. The use of efficient time integration methods has been determinant for integrating the short temporal scales exhibited by the flows at low $E$ and $\sigma$.

By means of numerical simulations by constraining the azimuthal symmetry, we have obtained the bifurcation diagrams of the oscillatory flows in terms of their physical properties and studied their dynamics. We have calculated branches of traveling waves as well as modulated waves with fixed azimuthal wave number from $m=4$ up to $m=7$. All the modulated waves are unstable except those with wave numbers $m=5,6$ in a small Rayleigh number 

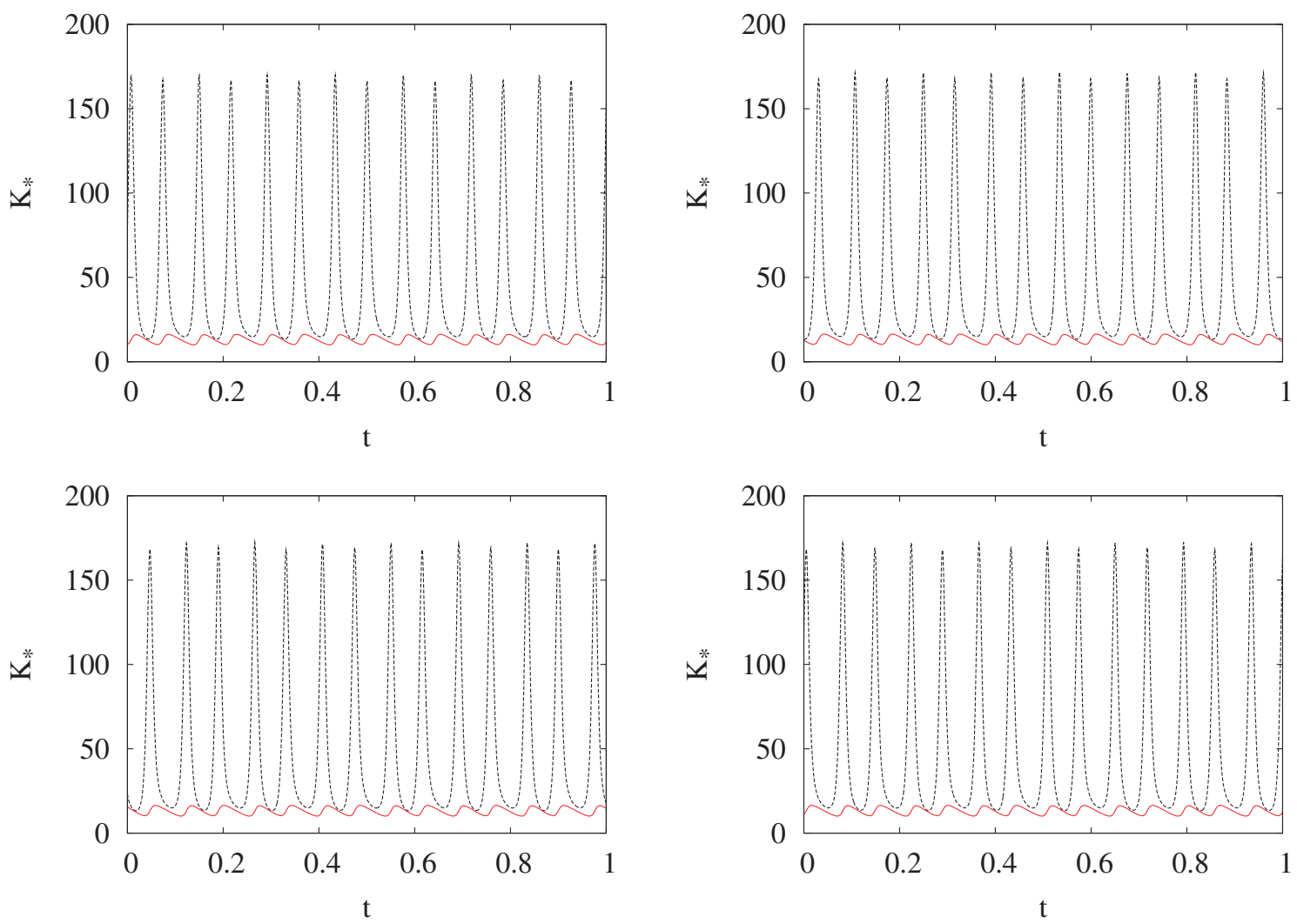

Figure 11. Time series of the axisymmetric (solid line) and nonaxisymmetric (dashed line) kinetic energy densities given by the low-dimensional model. The control parameters, from top to bottom and from left to right, are $\mu_{r}=900,904,905.43$, and 905.55. The rest of the parameters are $\alpha=250, \beta=10, \lambda=0.6$, and $\mu_{i}=100$.

interval. For these branches, and beyond the stability region, we have found a sequence of period doubling bifurcations of the modulation frequency leading to chaos. The periodicity and quasi periodicity of the solutions is quantified by means of an accurate analysis of the frequency spectrum provided by the Laskar algorithm. This algorithm, together with a five order time integration, allowed us to find resonant solutions.

The Poincaré sections have turned out to be useful in identifying the two-frequency solutions, resonances, and period doubling bifurcations. By adding a nonsymmetric perturbation to the solutions, we have studied their stability and found that the modulated waves are stable only in a small range of the parameter $R a$. For the latter solutions, convection is characterized by low heat transfer rates, and is strongly geostrophic. An abrupt increase of the zonal circulations occurs due the connection between the convective cells.

We have derived a five-degree model, based on the symmetries of the solutions, that reproduces with significative agreement the sequence of bifurcations and the type of solutions of the Boussinesq Navier-Stokes simulations. In this reduced model only two complex numbers, representing two modes with the same azimuthal symmetry but with different radial structure,

Copyright (C) by SIAM. Unauthorized reproduction of this article is prohibited. 

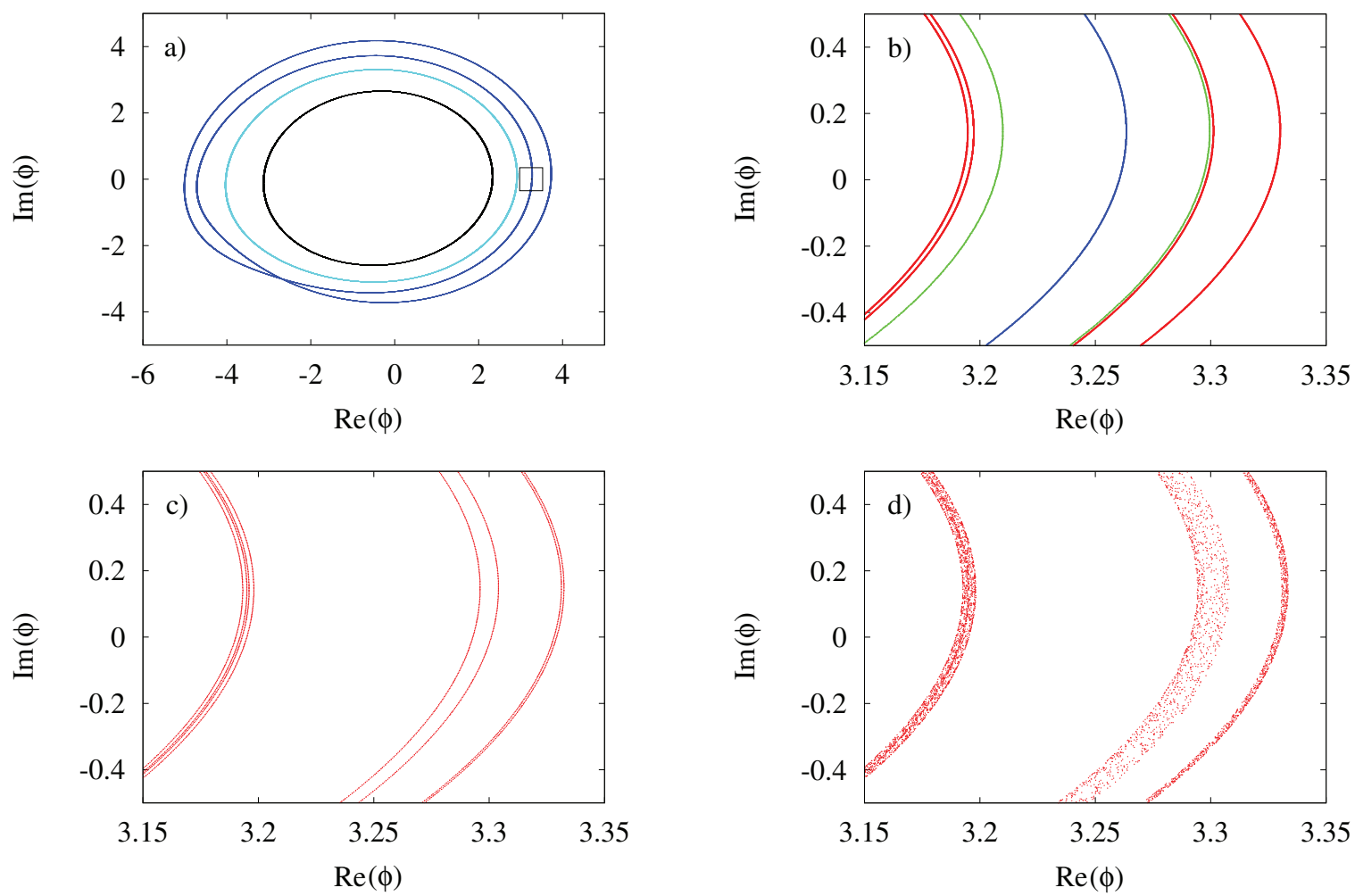

Figure 12. (a) Poincaré sections at $\Re(\psi)=0$ of $\Im(\phi)$ plotted versus $\Re(\phi)$. The control parameters from the inner to the outer curves are $\mu_{r}=679.423,750$, and 900 . In the outer curve (blue) the period of the modulation is doubled. (b) Detail of (a) (see the black box). The blue, green, and red curves correspond to the first, second, and third period doublings, respectively. The control parameters are $\mu_{r}=900,904$, and 905.43. (c) Solution corresponding to the fourth period doubling at $\mu_{r}=905.52$. (d) Chaotic solution at $\mu_{r}=905.55$.

together with a real number, which represents the axisymmetric mode $m=0$, are considered.

Acknowledgments. The authors wish to thank Stéphan Fauve for his helpful suggestions and comments when deriving the reduced model equations, and Carles Simó and anonymous reviewers for their useful advice.

\section{REFERENCES}

[1] F. M. Al-Shamali, M. H. Heimpel, And J. M. Aurnou, Varying the spherical shell geometry in rotating thermal convection, Geophys. Astrophys. Fluid Dyn., 98 (2004), pp. 153-169.

[2] M. Ardes, F. H. Busse, And J. Wicht, Thermal convection in rotating spherical shells, Phys. Earth Planet. Inter., 99 (1997), pp. 55-67.

[3] J. Aubert, N. Gillet, And P. Cardin, Quasigeostrophic models of convection in rotating spherical shells, Geochem. Geophys. Geosyst., 4 (2003), pp. 1052-1071.

[4] A. P. Bassom, A. M. Soward, And S. V. Starchenko, The onset of strongly localized thermal convection in rotating spherical shells, J. Fluid Mech., 689 (2011), pp. 376-416.

[5] F. H. Busse, Thermal instabilities in rapidly rotating systems, J. Fluid Mech., 44 (1970), pp. 441-460.

[6] P. CARDin And P. Olson, Chaotic thermal convection in rapidly rotating spherical shell: Consequences for flow in the outer core, Phys. Earth Planet. Inter., 82 (1994), pp. 235-239.

Copyright (c) by SIAM. Unauthorized reproduction of this article is prohibited. 
[7] P. Chossat and G. Iooss, The Couette-Taylor Problem, Springer-Verlag, New York, 1994.

[8] U. R. Christensen, Zonal flow driven by strongly supercritical convection in rotating spherical shells, J. Fluid Mech., 470 (2002), pp. 115-133.

[9] E. Dormy, A. M. Soward, C. A. Jones, D. Jault, and P. Cardin, The onset of thermal convection in rotating spherical shells, J. Fluid Mech., 501 (2004), pp. 43-70.

[10] R. E. Ecke, F. Zhong, AND E. KNOBLOCH, Hopf bifurcation with broken reflection symmetry in rotating Rayleigh-Bénard convection, Europhys. Lett., 19 (1992), pp. 177-182.

[11] F. Feudel, N. Seehafer, L. S. Tuckerman, and M. Gellert, Multistability in rotating spherical shell convection, Phys. Rev. E, 87 (2013), 023021.

[12] M. Frigo And S. G. Johnson, The design and implementation of FFTW3, Proc. IEEE, 93 (2005), pp. 216-231.

[13] F. GARCIA, Thermal Convection in Rotating Spherical Shells, Ph.D. thesis, Universitat Politècnica de Catalunya, Barcelona, Spain, 2012.

[14] F. Garcia, E. Dormy, J. SÁnchez, And M. Net, Two computational approaches for the simulation of fluid problems in rotating spherical shells, in Proceedings of the 5th International Conference on Computational Methods - ICCM2014, Vol. 1, G. R. Liu and Z. W. Guan, eds., Cambridge, England, 2014.

[15] F. Garcia, M. Net, B. García-Archilla, and J. SÁnchez, A comparison of high-order time integrators for thermal convection in rotating spherical shells, J. Comput. Phys., 229 (2010), pp. 7997-8010.

[16] F. Garcia, M. NET, AND J. SÁNChez, A comparison of high-order time integrators for highly supercritical thermal convection in rotating spherical shells, in Spectral and High Order Methods for Partial Differential Equations - ICOSAHOM 2012, Lect. Notes Comput. Sci. Eng. 95, M. Azaïez, H. El Fekih, and J. S. Hesthaven, eds., Springer International, Cham, Switzerland, 2014, pp. 273-284.

[17] F. Garcia, J. SÁnchez, And M. Net, Antisymmetric polar modes of thermal convection in rotating spherical fluid shells at high Taylor numbers, Phys. Rev. Lett., 101 (2008), 194501.

[18] F. GARCiA, J. SÁNChez, AND M. NET, Numerical simulations of thermal convection in rotating spherical shells under laboratory conditions, Phys. Earth Planet. Inter., 230 (2014), pp. 28-44.

[19] N. Gillet And C. A. Jones, The quasi-geostrophic model for rapidly rotating spherical convection outside the tangent cylinder, J. Fluid Mech., 554 (2006), pp. 343-369.

[20] K. Goto And R. A. VAn De Geisn, Anatomy of high-performance matrix multiplication, ACM Trans. Math. Software, 34 (2008), 12.

[21] C. A. Jones, Thermal and compositional convection in the outer core, in Core Dynamics, Treatise on Geophysics, Vol. 8, Elsevier, Amsterdam, 2007, pp. 131-185.

[22] K. Kimura, S. Takehiro, and M. Yamada, Stability and bifurcation diagram of Boussinesq thermal convection in a moderately rotating spherical shell, Phys. Fluids, 23 (2011), 074101.

[23] J. LASKAR, Frequency analysis of a dynamical system, Celest. Mech. Dynam. Astron., 56 (1993), pp. 191196.

[24] G. M. Lewis And W. NAGATA, Double Hopf bifurcations in the differentially heated rotating annulus, SIAM J. Appl. Math., 63 (2003), pp. 1029-1055.

[25] X. Liao, K. Zhang, and T. Feng, On thermal instabilities and differential rotation in rapidly rotating stars: A new quasi-geostrophic approximation, Astrophys. J., 631 (2005), pp. 518-528.

[26] V. Morin And E. Dormy, Time dependent $\beta$-convection in rapidly rotating spherical shells, Phys. Fluids, 16 (2004), pp. 1603-1609.

[27] V. Morin And E. Dormy, Dissipation mechanisms for convection in rapidly rotating spheres and the formation of banded structures, Phys. Fluids, 18 (2006), 068104.

[28] M. Net, F. Garcia, And J. SÁnchez, On the onset of low-Prandtl-number convection in rotating spherical shells: Non-slip boundary conditions, J. Fluid Mech., 601 (2008), pp. 317-337.

[29] P. Olson, Laboratory experiments on the dynamics of the core, Phys. Earth Planet. Inter., 187 (2011), pp. $1-18$.

[30] F. PÉtrélis and S. Fauve, Mechanisms for magnetic field reversals, Philos. Trans. R. Soc. Lond. Ser. A, 368 (2010), pp. 1595-1605.

[31] F. Pétrélis, S. Fauve, E. Dormy, and J. P. Valet, Simple mechanism for reversals of Earth's magnetic field, Phys. Rev. Lett., 102 (2009), 144503.

[32] E. Plaut And F. H. Busse, Multicellular convection in rotating annuli, J. Fluid Mech., 528 (2005), pp. 119-133.

Copyright (C) by SIAM. Unauthorized reproduction of this article is prohibited. 
[33] P. H. Roberts, On the thermal instability of a rotating fluid sphere containing heat sources, Philos. Trans. R. Soc. Lond. Ser. A, 263 (1968), pp. 93-117.

[34] J. SÁnchez, F. GARCIA, AND M. NET, Computation of azimuthal waves and their stability in thermal convection in rotating spherical shells with application to the study of a double-Hopf bifurcation, Phys. Rev. E, 87 (2013), 033014.

[35] J. SÁnchez, M. NET, AND J. M. VEGA, Amplitude equations close to a triple- $(+1)$ bifurcation point of $\mathbb{D}_{4}$-symmetric periodic orbits in $\mathbb{O}(2)$-equivariant systems, Discrete Contin. Dyn. Syst. Ser. B, 6 (2006), pp. 1357-1380.

[36] R. Simitev and F. H. Busse, Patterns of convection in rotating spherical shells, New J. Phys., 5 (2003), pp. 97.1-97.20.

[37] A. M. SOWARD, On the finite amplitude thermal instability in a rapidly rotating fluid sphere, Geophys. Astrophys. Fluid Dyn., 9 (1977), pp. 19-74.

[38] A. Tilgner and F. H. Busse, Finite amplitude convection in rotating spherical fluid shells, J. Fluid Mech., 332 (1997), pp. 359-376.

[39] K. Zhang, Spiralling columnar convection in rapidly rotating spherical fluid shells, J. Fluid Mech., 236 (1992), pp. 535-556.

[40] K. Zhang, X. LiaO, And F. H. Busse, Asymptotic solutions of convection in rapidly rotating non-slip spheres, J. Fluid Mech., 578 (2007), pp. 371-380.

[41] K. Zhang, X. LiaO, And F. H. Busse, Asymptotic theory of inertial convection in a rotating cylinder, J. Fluid Mech., 575 (2007), pp. 449-471.

[42] K. K. ZhANG, Convection in a rapidly rotating spherical shell at infinite Prandtl number: Transition to vacillating flows, Phys. Earth Planet. Inter., 72 (1992), pp. 236-248.

Copyright $\odot$ by SIAM. Unauthorized reproduction of this article is prohibited. 